\title{
A Study on the Intraocular Pressure of the Affected and Unaffected Eyes in Patients with Isolated Cranial Nerve Palsies
}

\author{
Özlem Dikmetaşs, , [MD] \\ ORCID:0000-0001-5670-2384 \\ Bogomil Voykov', [MD] \\ ORCID:0000-0002-1706-126X
}

${ }^{1}$ Department of Ophthalmology, Hacettepe University School of Medicine, Ankara, Turkey.

${ }^{2}$ University Eye Hospital, Eberhard-Karls University, Tuebingen, Germany.

Corresponding Author: Ozlem Dikmetas, MD

Department of Ophthalmology, Hacettepe University School of Medicine, Ankara, Turkey.

e-mail: ozlemdikmetas@gmail.com

Telephone: +905354700829

DOI: $10.32552 / 0$. ActaMedica.422

\section{we) ABSTRACT Cen}

Objective: The role of elevated intraocular pressure in the progression of glaucoma is well known. The exact mechanisms of intraocular pressure regulation are still unclear. Central control has been suggested, but the autonomic pathway through which it acts is not known. The aim of this study was to investigate if isolated cranial nerve palsies of the third, fourth and sixth nerves are associated with an intraocular pressure difference between the affected and the unaffected eyes.

Materials and Methods: This was a retrospective study including patients diagnosed with a third, fourth and sixth nerve palsy at a single tertiary centre. We included only patients with an isolated unilateral palsy. Patients with a history of strabismus, orbital disease or neurosurgical cases were excluded.

Results: The charts of 1712 patients were reviewed. Third, fourth and sixth nerve palsies were found in 469 patients, 314 patients and 929 patients, respectively. Of all patients, 190 (10.6\%) were eligible for inclusion in the study. A third nerve, fourth nerve or sixth nerve palsy was present in $85(44.7 \%), 65(34.2 \%)$ and 40 (21.1\%) patients, respectively. The mean intraocular pressure of the affected eyes and the unaffected eyes was not statistically significant different: $14.1 \pm 3.1 \mathrm{mmHg}$ vs. $14.6 \pm 2.7 \mathrm{mmHg}$ in the cranial nerve 3 group ( $\mathrm{p}=0.087) ; 13.6 \pm 2.6 \mathrm{mmHg}$ vs. $13.7 \pm 2.3 \mathrm{mmHg}$ in the cranial nerve 4 group $(\mathrm{p}=0.69)$; and $14.3 \pm 2.7 \mathrm{mmHg}$ vs. $14.9 \pm 3.3 \mathrm{mmHg}$ in the cranial nerve 6 group ( $\mathrm{p}=0.089$ ). There was no statistically significant difference between the mean intraocular pressure differences of the affected and unaffected eyes among the three groups $(\mathrm{p}=0.47)$.

Conclusion: Our study demonstrated no difference in intraocular pressure between affected and unaffected eyes in patients with an isolated cranial nerve palsy. These findings are the first and important for ophthalmology practice.

Key Words: third cranial nerve palsy, fourth cranial nerve palsy, sixth cranial nerve palsy, intraocular pressure, autonomic nervous system

Received: 01 January 2020, Accepted: 10 March 2020

Published online: 17 March 2020

\section{INTRODUCTION}

The role of elevated intraocular pressure (IOP) in the incidence and progression of glaucoma is well known [1-4]. However, the exact mechanisms of IOP regulation are still unclear. The effect of extra ocular muscles' tone on IOP is not known exactly. Circadian variations in IOP are associated with human circadian rhythms, which are under the control of the autonomic nervous system $[5,6]$. Also, a phenomenon whereby the change of IOP in one eye leads to a corresponding IOP alteration in the contralateral eye, the "ophthalmotonic consensual reaction" (OCR), suggests an involvement of the autonomic nervous system in the control of IOP. Therefore, the question arises which afferent and efferent pathways and central nervous centres are involved in the regulation of IOP. The aim of this study was to investigate if isolated cranial nerve $(\mathrm{CN})$ palsies of the third, fourth and sixth nerves are associated with an IOP difference between the affected and the unaffected eye.. 


\section{MATERIALS and METHODS}

This was a retrospective study including patients diagnosed with a third, fourth and sixth nerve palsy at a single tertiary centre. We included only patients with an isolated unilateral palsy, in one week after presentation to ophthalmology clinic. Diagnosis was confirmed in a neuroophthalmological examination including visual field testing, ocular motility, pupil examination and fundus examination. Other inclusion criteria were existing IOP measurements at the time of presentation and at least three times at different daily hours $(08.00,14.00,18.00)$, with a single same technician, best-corrected visual acuity, slit-lamp anterior segment and fundus examination, and orthoptic status in both eyes. Patients with a history of strabismus, orbital disease, trauma, trigeminal nerve palsy, optic neuropathy, giant cell arteritis or neurosurgical cases were excluded from the study. The following data were collected for each patient: age, sex, date of presentation, clinical symptoms and ophthalmic history, surgical and medical management, best-corrected visual acuity (BCVA) and IOP. Glaucoma patients are controlled by medications at least for a year. Diabetic patients who don't have any neurological symptoms are well controlled. The statistical analysis was performed with the JMP 14.0 statistical package (SAS Institute Inc., Cary, NC, USA). The t test was used to compare continuous variables. The mean difference in IOP between affected and unaffected eyes for the three groups was compared using one-way analysis of variance (ANOVA). A value of $p<0.05$ was considered statistically significant. This study was approved by the Institutional Review Board of the University Eye Hospital, Eberhard-Karls University, Tuebingen, Germany (740/2017B02-10.11.2017). This study adhered to the tenets of the Declaration of Helsinki.

\section{RESULTS}

The charts of 1712 patients were reviewed. Third, fourth and sixth nerve palsies were found in 469 patients, 314 patients and 929 patients, respectively.
Of all patients, 190 (10.6\%) were eligible for inclusion in the study. Demographic data of the patients are presented in Table 1. A third nerve, fourth nerve or sixth nerve palsy was present in 85 (44.7\%), 65 (34.2\%) and 40 (21.1\%) patients, respectively. Diabetes mellitus was thought to cause the palsy in $12.4 \%$ of the eyes in the 3.CN palsy group, $1.5 \%$ of the eyes in the $4 . \mathrm{CN}$ palsy group and $20 \%$ of the eyes in the 6.CN palsy group. Systemic hypertension was reported by $31.9 \%, 41.5 \%$, and none in the 3.CN, 4.CN and 6.CN palsy groups, respectively.

In all three groups the mean IOP of the affected eyes was lower than that of the unaffected eyes: $14.1 \pm 3.1$ $\mathrm{mmHg}$ vs. $14.6 \pm 2.7 \mathrm{mmHg}$ in the CN3 Group; 13.6 $\pm 2.6 \mathrm{mmHg}$ vs. $13.7 \pm 2.3 \mathrm{mmHg}$ in the CN4 Group; and $14.3 \pm 2.7 \mathrm{mmHg}$ vs. $14.9 \pm 3.3 \mathrm{mmHg}$ in the CN6 Group. However, the differences were small and not statistically significant: $-0.39 \pm 2.1(95 \% \mathrm{Cl}-0.8-0.06$; $\mathrm{p}=0.087) ;-0.11 \pm 2.2(95 \% \mathrm{Cl}-0.65-0.43 ; \mathrm{p}=0.69)$; and $-0.63 \pm 2.3(95 \% \mathrm{Cl}-1.35-0.1 ; p=0.089)$ for the CN3, CN4 and CN6 Groups, respectively. There was no statistically significant difference between the mean IOP differences of the affected and unaffected eyes among the three groups $(p=0.47)$.

\section{DISCUSSION}

Increased intraocular pressure is considered to be the most important risk factor for the incidence and progression of glaucoma [4, 7-9]. An imbalance between aqueous humor secretion in the ciliary body $(\mathrm{CB})$ and outflow in the anterior chamber angle mainly due to increased outflow resistance is responsible for the increased IOP in glaucoma [10, 11]. However, little is known about the exact mechanisms of IOP regulation. Theoretically, IOP can be influenced by changes in the aqueous humor production in the ciliary processes and/or alteration of the outflow facility involving the trabecular meshwork, the Schlemm's canal, the collector channels and the episcleral blood vessels. All of these structures are densely innervated by sympathetic, parasympathetic, sensory and intrinsic neurons suggesting a role of the autonomic nervous system in the regulation of the IOP (Figure 1). 
Table 1. Distribution of AST, ALT, HBV DNA values 1st, 3rd, 6th, 9th and 12th month

\begin{tabular}{|c|c|c|c|}
\hline & 3.CN PALSY & 4.CN PALSY & 6.CN PALSY \\
\hline Number of Patients & 97 (40\%) & 65 (27\%) & 40 (16\%) \\
\hline Mean Age (Range) & $67.7 \pm 18.8(13-100)$ & $61.9 \pm 16.8(26-96)$ & $65.7 \pm 18.3(22-101)$ \\
\hline Right Eye & $46(47.4 \%)$ & 37 (56.9\%) & $24(60 \%)$ \\
\hline Left Eye & $51(52.6 \%)$ & $28(43.1 \%)$ & $16(40 \%)$ \\
\hline Females & $50(51.5 \%)$ & $30(46.2 \%)$ & $18(45 \%)$ \\
\hline Males & 47 (48.5\%) & 35 (53.8\%) & 22 (55\%) \\
\hline IOP Affected Eye & $14.3 \pm 3.1(5-24)$ & $13.6 \pm 2.6(8-23)$ & $14.3 \pm 2.7(8-20)$ \\
\hline IOP Unaffected Eye & $14.7 \pm 2.8(9-24)$ & $13.7 \pm 2.3(10-20)$ & $14.9 \pm 3.3(8-23)$ \\
\hline BCVA RE (logMAR) & $0.2 \pm 0.4(0-2.3)$ & $0.04 \pm 0.08(0-0.3)$ & $0.17 \pm 0.39(0-2.3)$ \\
\hline BCVA LE (logMAR) & $0.31 \pm 0.61(0-2.3)$ & $0.06 \pm 0.10(0-0.4)$ & $0.19 \pm 0.50(0-2.30)$ \\
\hline CDR RE & $0.32 \pm 0.21(0.1-1)$ & $0.33 \pm 0.15(0.1-0.8)$ & $0.39 \pm 0.18(0.2-1)$ \\
\hline CDR LE & $0.34 \pm 0.18(0.1-1)$ & $0.32 \pm 0.14(0.1-0.8)$ & $0.36 \pm 0.16(0.1-0.8)$ \\
\hline Glaucoma & $10(10.3 \%)$ & $5(7.7 \%)$ & $6(15 \%)$ \\
\hline Hypertension & 29 (31.9\%) & 27 (41.5\%) & $0(0 \%)$ \\
\hline Diabetes mellitus & 12 (12.4\%) & 1 (1.5\%) & 8 (20\%) \\
\hline
\end{tabular}

CN: cranial nerve, BCVA: best corrected visual acuity, IOP: intraocular pressure, CDR: cup disc ratio, RE: right eye, LE: left eye

\section{Regulation of IOP}

\section{Aqueous humor formation}

\section{Aqueous humor outflow}

\section{Ciliary body}

\section{CB blood vessels Ciliary epithelium Trabecular meshwork Episcleral vessels Scleral spur}

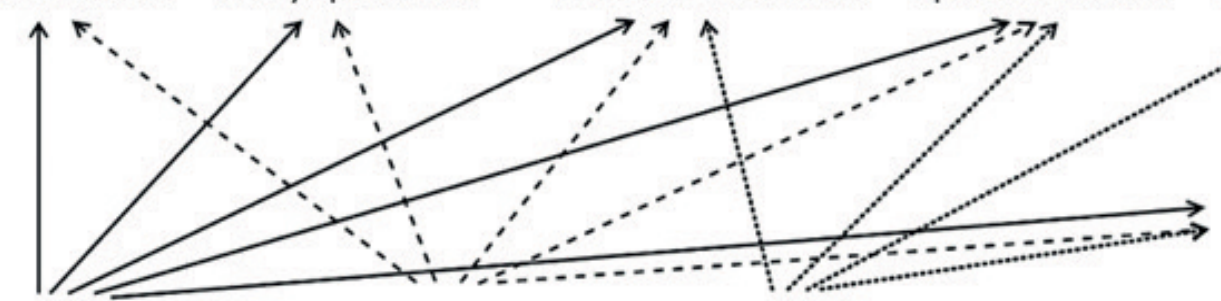

\section{Choroid}

\section{CG}

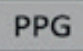

PPG

$\uparrow$

N. III

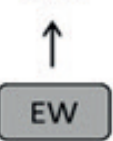

N. VII

Parasympathetic

\section{SCG}

TG

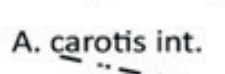

$\uparrow \cdots$

ILM

Sympathetic

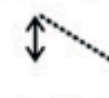

N. V

Sensory

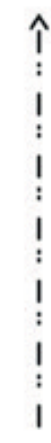

Intrinsic choroidal neurons

Figure 1. A schematic diagram showing the autonomic innervation of intraocular structures possibly involved in the IOP regulation. 
CG- Ciliary ganglion; EW- Edinger-Westphal nucleus; ILM- Intermediolateral nucleus; PPGPterygopalatine ganglion; SCG- Superior cervical ganglion; SSN- Superior salivatory nucleus; TGTrigeminal ganglion; TN- Trigeminal nucleus

\section{Aqueous Humor Secretion}

A detailed review of the autonomic control of the IOP has been presented by McDougal and Gamlin [5]. Briefly, the ciliary body vasculature receives parasympathetic innervation along the facial nerve by fibers arising from cholinergic, postganglionic neurons in the pterygopalatine ganglion. It has been suggested that decreased parasympathetic innervation might be expected to reduce blood flow in the ciliary body and aqueous humor production, and hence reduce IOP. In accordance to the latter, Ruskell et al. demonstrated a long-lasting reduction in IOP in monkeys after the removal of the pterygopalatine ganglion [12]. Sympathetic innervation of the ciliary body vasculature is provided by fibers that arise from noradrenergic postganglionic neurons in the superior cervical ganglion [13]. Increased sympathetic innervation results in decreased ciliary body blood flow with a consequent reduction of aqueous humor secretion and decrease in IOP [1416]. Also, the trigeminal nerve provides innervation of ciliary body blood vessels. Its stimulation causes increased blood flow in the ciliary body and significantly increased IOP [17]. Additionally, non-pigmented epithelial cells in the ciliary epithelium possess adrenergic and muscarinic receptors suggesting a possible direct control of the autonomic nervous system on the secretion of aqueous humor [18, 19].

\section{Outflow Facility}

A major part of the aqueous humor leaves the eye via the conventional route in the anterior chamber angle. Accommodation or stimulation of the third cranial nerve causes a contraction of the ciliary muscle which is followed by changes in the trabecular meshwork and possibly dilation of Schlemm's canal leading to a decreased outflow resistance [20]. Also, studies have shown sympathetic, parasympathetic and sensitive innervation of the trabecular meshwork and scleral spur region [21-23].

The episcleral veins are another important part of the outflow system of the eye. They are innervated by sympathetic, parasympathetic and trigeminal nerve fibres [24]. It has been suggested that the episcleral circulation is under tonic neural control that is either arterial (vasodilatory) or venous (vasoconstrictor) [25].

Taken together the above findings suggest a possible role of the third, fifth and seventh cranial nerves in the control of IOP. The fourth and the sixth cranial nerves are not involved in the autonomic control of the eye. Consequently, we did not expect to find a difference in IOP between affected and unaffected eyes in patients with unilateral isolated fourth and sixth cranial nerve palsy. The third cranial nerve is involved in the autonomic regulation of the sphincter pupillae and the ciliary muscle and, at least theoretically, in the neural control of IOP. However, we did not find any difference between the IOP of the affected and unaffected eyes in patients with an isolated third cranial nerve palsy. There are several possible explanations for these results. Firstly, due to the retrospective nature of our study only ten percent of the patients were eligible for inclusion mainly because of missing IOP measurements in one or both eyes at the time of presentation in the majority of cases. Secondly, there is no data concerning the latency between a triggering event causing a change in the IOP homeostasis and the response of the regulatory mechanisms to that change. $A$ baroreflex-type of response could be expected to occur within seconds similarly to the baroreflex dealing with postural hypotension. However, slowly developing and long-lasting adaptive changes are also possible. Consequently, even if a third cranial nerve palsy is immediately followed by a change of IOP in the affected eye, it is possible that homeostasis is re-established after some time. Since we measured IOP at the time of presentation, which could have been days or even weeks after occurrence of the palsy, we might have simply missed the time at which an IOP difference between the affected and unaffected eyes would have been present. Consistent with that possibility, studies on the ophthalmotonic 
consensual reaction (OCR) suggest, that changes in IOP might be better observed shortly after a triggering event than after weeks or months $[26,27]$.

Finally, the exact location or even the existence of a central control of IOP is still unclear. Some early studies in the 1950s and 1960s suggested a diencephalic control of the IOP in cats [28-30]. However, electrical stimulation of the diencephalon caused concurrent changes in arterial mean pressure as well and their findings were not broadly accepted. More recently, the role of dorsomedial and perifornical hypothalamus in the control of IOP has been proposed [31]. However, it is not clear through which autonomic pathway it acts. It has been shown that stimulation of the Edinger-Westphal nucleus can result in increased IOP in cats suggesting the involvement of the third cranial nerve in this process [32]. The exact mechanisms are, however, still unknown and may include increased extraocular muscle tone or increased intraocular blood flow. Other studies have reported that electrical stimulation of the superior salivatory nucleus significantly increases both episcleral venous pressure and IOP, suggesting a role of the seventh cranial nerve in the regulation of IOP [33].

In conclusion, our study demonstrated no difference in IOP between affected and unaffected eyes in patients with isolated third, fourth or sixth cranial nerve palsy. This is the first study about cranial nerve palsies and intraocular pressure. More research is needed into the central control of IOP and the exact mechanisms through which IOP is regulated.

\section{CONFUCT Of INTEREST STATEMENT}

None of the authors has conflict of interest with the submission. 
[1] Quigley HA. Glaucoma. Lancet 2011;377:1367-77.

[2] Anderson DR. Glaucoma: the damage caused by pressure. XLVI Edward Jackson memorial lecture. Am J Ophthalmol. 1989;108:485-95.

[3] Davis BM, Crawley L, Pahlitzsch M, et al. Glaucoma: the retina and beyond. Acta Neuropathol 2016;132:807-826.

[4] Musch DC, Gillespie BW, Lichter PR, et al. Visual field progression in the Collaborative Initial Glaucoma Treatment Study the impact of treatment and other baseline factors. Ophthalmology 2009;116:200-7.

[5] McDougal DH, Gamlin PD. Autonomic control of the eye. Compr Physiol 2015;5:439-73.

[6] Bremner F. Pupil evaluation as a test for autonomic disorders. Clin Auton Res 2009;19:88-101.

[7] Heijl A, Leske MC, Bengtsson B, et al. Reduction of intraocular pressure and glaucoma progression: results from the Early Manifest Glaucoma Trial. Arch Ophthalmol. 2002;120:1268-79.

[8] Guo ZZ, Chang K, Wei X. Intraocular pressure fluctuation and the risk of glaucomatous damage deterioration: a Meta-analysis. Int J Ophthalmol 2019;12:123-128.

[9] Matlach J, Bender S, Konig J, et al. Investigation of intraocular pressure fluctuation as a risk factor of glaucoma progression. Clin Ophthalmol 2019;13:9-16.

[10] O'Callaghan J, Cassidy PS, Humphries P. Open-angle glaucoma: therapeutically targeting the extracellular matrix of the conventional outflow pathway. Expert Opin Ther Targets 2017;21:1037-1050.

[11] Stamer WD, Braakman ST, Zhou EH, et al. Biomechanics of Schlemm's canal endothelium and intraocular pressure reduction. Prog Retin Eye Res 2015;44:86-98.

[12] Ruskell GL. An ocular parasympathetic nerve pathway of facial nerve origin and its influence on intraocular pressure. Exp Eye Res 1970;10:319-30.

[13] Stone RA, Tervo T, Tervo K, et al. Vasoactive intestinal polypeptide-like immunoreactive nerves to the human eye. Acta Ophthalmol (Copenh) 1986;64:12-8.

[14] Belmonte C, Bartels SP, Liu JH, et al. Effects of stimulation of the ocular sympathetic nerves on IOP and aqueous humor flow. Invest Ophthalmol Vis Sci 1987;28:1649-54.

[15] Greaves DP, Perkins ES. Influence of the sympathetic nervous system on the intra-ocular pressure and vascular circulation of the eye. Br J Ophthalmol 1952;36:258-64.

[16] Uusitalo R. Effect of sympathetic and parasympathetic stimulation on the secretion and outflow of aqueous humour in the rabbit eye. Acta Physiol Scand 1972;86:315-26.

[17] Stjernschantz J, Geijer C, Bill A. Electrical stimulation of the fifth cranial nerve in rabbits: effects on ocular blood flow, extravascular albumin content and intraocular pressure. Exp Eye Res 1979;28:229-38.

[18] Gupta N, McAllister R, Drance SM, et al. Muscarinic receptor M1 and M2 subtypes in the human eye: QNB, pirenzipine, oxotremorine, and AFDX-116 in vitro autoradiography. Br J Ophthalmol 1994;78:555-9.

[19] Polansky JR, Zlock D, Brasier A, et al. Adrenergic and cholinergic receptors in isolated non-pigmented ciliary epithelial cells. Curr Eye Res 1985;4:517-22.

[20] B'Ann True Gabelt PLK. Production and Flow of Aqueous humor. In: Levin LK, PL, , editor. Adler's physiology of the Eye: Clinical Application New York: Elsevier, 2011.

[21] Nomura T, Smelser GK. The identification of adrenergic and cholinergic nerve endings in the trabecular meshwork. Invest Ophthalmol 1974;13:525-32.

[22] Selbach JM, Gottanka J, Wittmann M, et al. Efferent and afferent innervation of primate trabecular meshwork and scleral spur. Invest Ophthalmol Vis Sci 2000;41:2184-91.

[23] Thieme H, Hildebrandt J, Choritz L, et al. Muscarinic receptors of the M2 subtype in human and bovine trabecular meshwork. Graefes Arch Clin Exp Ophthalmol 2001;239:310-5.

[24] Selbach JM, Rohen JW, Steuhl KP, et al. Angioarchitecture and innervation of the primate anterior episclera. Curr Eye Res 2005;30:337-44.

[25] Zamora DO, Kiel JW. Topical proparacaine and episcleral venous pressure in the rabbit. Invest Ophthalmol Vis Sci 2009;50:2949-52.

[26] Voykov B, Blumenstock G, Ziemssen F, et al. Fluctuations of the Intraocular Pressure in One Eye Influence the Intraocular Pressure in the Fellow Eye. Curr Eye Res 2017;42:211-216.

[27] Radcliffe NM, Musch DC, Niziol LM, et al. The effect of trabeculectomy on intraocular pressure of the untreated fellow eye in the collaborative initial glaucoma treatment study. Ophthalmology 2010;117:2055-60.

[28] Gloster J. Responses of the intra-ocular pressure to diencephalic stimulation. Br J Ophthalmol. 1960;44:649-64.

[29] Gloster J, Greaves DP. Effect of diencephalic stimulation upon intra-ocular pressure. $\mathrm{Br} \mathrm{J}$ Ophthalmol 1957;41:513-32.

[30] Von Sallmann L, Lowenstein O. Responses of intraocular pressure, blood pressure, and cutaneous vessels to electric stimulation in the diencephalon: the ninth Francis I. Proctor Lecture. Am J Ophthalmol 1955;39:11-29.

[31] Samuels BC, Hammes NM, Johnson PL, et al. Dorsomedial/ Perifornical hypothalamic stimulation increases intraocular pressure, intracranial pressure, and the translaminar pressure gradient. Invest Ophthalmol Vis Sci.2012;53:7328-35.

[32] Gherezghiher T, Hey JA, Koss MC. Parasympathetic nervous control of intraocular pressure. Exp Eye Res.1990;50:457-62.

[33] Strohmaier CA, Reitsamer HA, Kiel JW. Episcleral venous pressure and IOP responses to central electrical stimulation in the rat. Invest Ophthalmol Vis Sci 2013;54:6860-6. 